\title{
Space Applications for Input Pulse Shaping Filter
}

\author{
D. Venkata Ramana, S. Pal \\ ISRO Satellite Centre \\ \& \\ A.P. Shiva Prasad \\ Indian Institute d Science
}

\begin{abstract}
A pulse-shaping technique superior to various other existing pulse shaping techniques has been suggested. A practical way of implementing the scheme has been discussed and the results found to be encouraging. A new Spike Suppression (SS). Window function has also been suggested for obtaining low side lobe levels and to suppress spikes in phase shift keyingspectrumsthat exists at high bit rate data transmission. The characteristics such as low side lobe levels and spike eliminationhave been achievedusing new techniques that are essential for space applications.
\end{abstract}

\section{INTRODUCTION}

th continuing growth of communications and increasing numbers of users, frequency bands are becoming more and more congested. In order to cope with this frequency congestion, methods to increase bandwidth utilization have been studied $[1,2]$. These methods consider efficient modulation, which is implemented using pulse-shaping methods. Two kinds of pulse-shaping methods have been investigated to realize bandwidth efficiency. One of the pulse-shaping methods is pre-modulation pulse-shaping which performs a low pass filtering before modulation to reduce bandwidth. The other method is post-modulation pulse-shaping which is band pass filtering after modulation. Many authors have investigatedpre-modulation pulse-shaping and post-modulation pulse shaping with various filters. Pre-modulation pulse shaping can induce spikes and results in adjacent channel interference: post-modulation pulse-shaping can induce a non-constant envelope signaling which can spread the reduced bandwidth.

Several papers have been presented on various modulation schemes $[1,2]$. The selection of a particular optimum

Authors' Current Addresses:

D.V. Ramana, S. Pal, Digital and Communication Area, ISRO Satellite Centre, Bangalore560 017, India; and A.P. Shiva Prasad, Department of ECE, Indian Institute of Science, Bangalore 560 012, India

Manuscript received April 22, 2004; revised May 14 and June 3, 2004.

$0885 / 8985 / 0 \$ / \$ 17.0002005$ IEEE modulation technique depends upon the specific system configuration. The system parameters of importance are: 1)the Bandwidth and Time (BT) product that describes the bandwidth efficiency; 2) adjacent channel constraints; 3) co-channel interference; 4) tolerable total $\mathrm{E}_{\mathrm{bo}} / \mathrm{N}_{\mathrm{o}}$ degradation; 5) the design of the synchronization circuitry; and 6 ) finally, the specific characteristics of the filters used. MSS is preferred for wider-band applications where BT is greater than one, whereas QPSS is the best for the very narrowband situation. In this paper, QPSS modulation scheme has been considered since it is optimum modulation technique - both in terms of power and bandwidth for data transmission from space to ground.

The demand for transmission of large data through band-limited channels bas been increasing rapidly. Since availability of spectrum is at premium, need for bandwidth efficient modulation schemes have driven a great deal of research in this area. One such method for bandwidth efficiency is pulse-shaping or windowing. By using window function, one can reduce the power contained in the side lobes and concentrate the signal near the carrier.

In general, the MPSS spectrum consists of a main lobe representing the middle of the spectrumand various sidelobes located on either side of the main lobe. Shaping the spectrum should satisfy two criteria [3] i.e,; 1), the main lobe should be as narrow as possible, 2) the maximum side lobe level should be as small as possible relative to the main lobe.

In recent years, studies have shown that Phase Shift Keying (PSK) modulation is particularly suited to digital satellite communications. The power spectra of a PSK signal has a $[\sin x / x]^{2}$ characteristic that may interfere with adjacent channels. To suppress the out-of-band interference, it may be necessary to remove the side lobes by filtering at the transmitter. Two ways to reduce the PSK modulations spectrum are pre-modulation filtering and post modulation filtering $[1,2]$. Post-modulation filtering requires a narrow-band filter at the Radio Frequency (RF) following the modulator output. For direct RF carrier modulation requirements, phase equalized post-modulation filtering with low and medium (less than 20MBPS) data rates become very complex. Hence, pre-modulation filtering can be used instead, provided that the modulator is linear. 


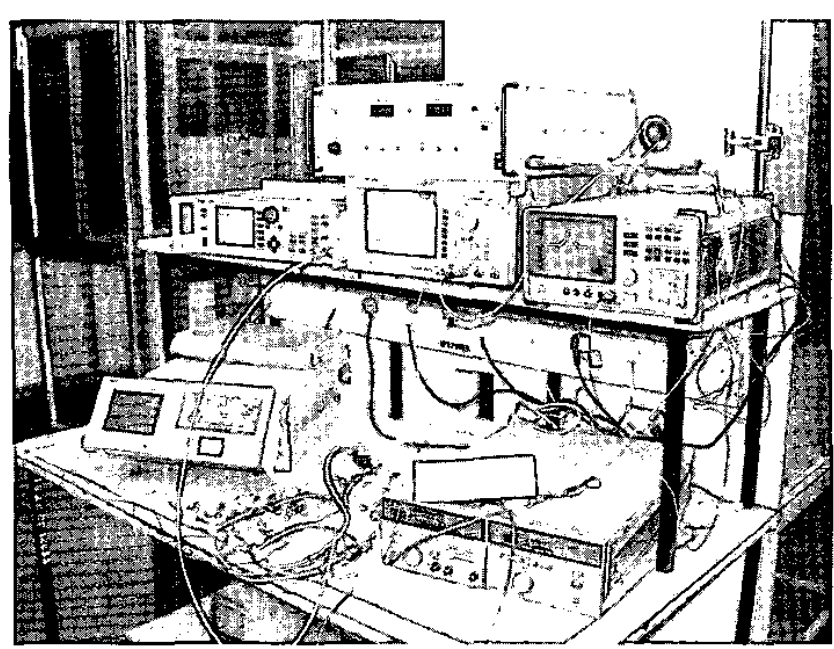

Fig. 1. Photograph of experimental set-up

Development of bandwidth and power efficient modulation schemes resulted in quadrature carrier modulation techniques. Digital phase modulation that allows transmitting discrete phase angles is more popular and easily adaptable. Among the PSK systems that create discrete phase states on the carrier keeping the envelope of the carrier constant, Quadri Phase Shift Keying (QPSK) is the most commonly adopted and is popular due to its relative ease of realization and optimum performance - in terms of power as well-as bandwidth. The QPSK modulation is perhaps the best trade-off between power and bandwidth requirements among all other modulation schemes [4].

Post-modulation filtering or pre-modulation filtering of PSK signals, however, produces a time-varying envelope and intersymhol interference (ISI). When such a filtered signal is transmitted through a nonlinear channel, such as Travelling Wave Tube Amplifier (TWTA) operating in its most efficient mode, i.e., close to saturation, the filtered side lobes are regenerated. This is caused by the non-linear input-output power (AM-to-AM conversion) characteristics of the channel [5]. The regenerated side lobes produce adjacent channel interference, and hence cause transmission impairments into the neighboring channels. Consequently, it is important to produce a hand limited PSK signal with a constant or nearly constant envelope.

In most wireless communication systems, spectrum would be too wide to meet Frequency Coordinate Committee (FCC) regulations and would also cause unwanted interference by spilling into adjacent channels. Placing a very narrow-band pass filter on the output of the modulator could narrow the spectrum. However, this is usually not practical since the Q of the filter would have to he very high. This is due to the fact that the transmitted carrier frequency is usually much higher than the data rate. Such a high Q filter would be difficult to build, expensive, and would cause significant distortion on the transmitted waveform due to rapid phase variations at the band edges [6], A better way to limit the output spectrum is by filtering the base band I and Q modulation signals before

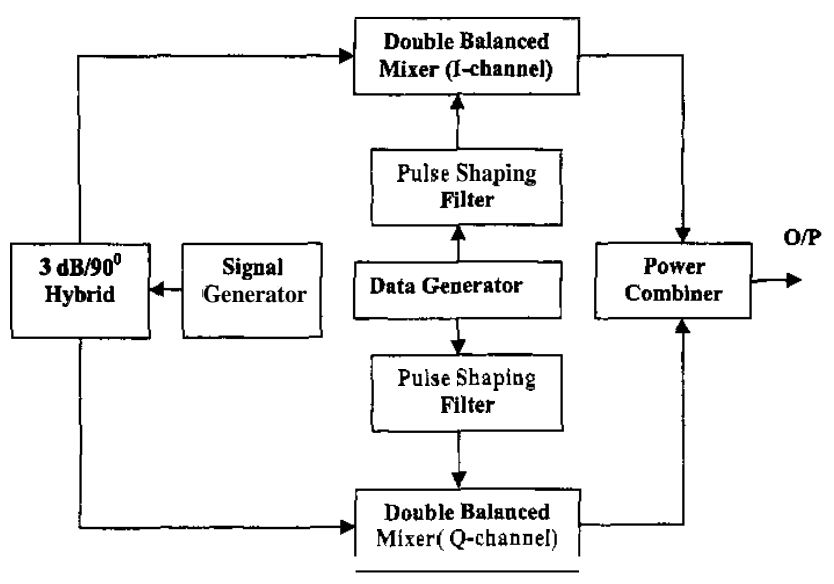

Fig. 2. Block diagram of the hardware set-up

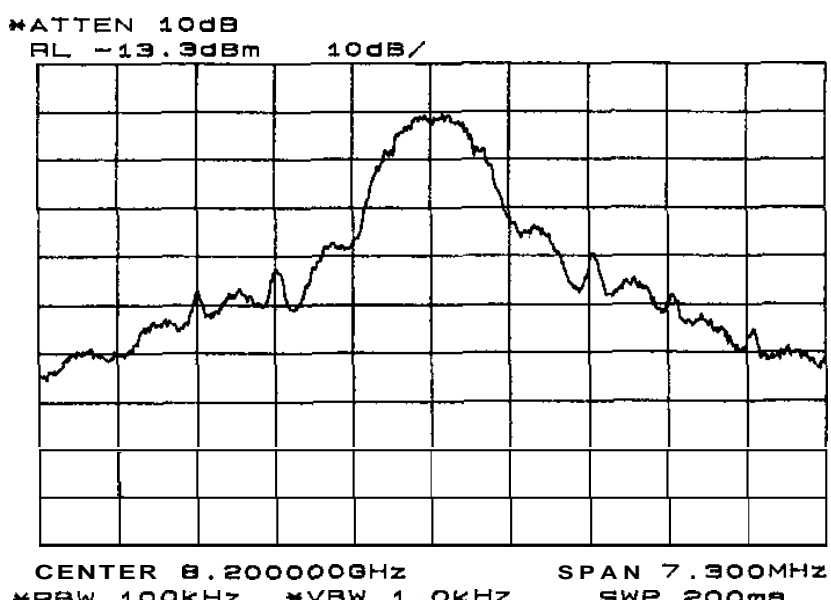

\#REW $100 \mathrm{KHZ}$ \#BW 1.0KHZ

SWP $200 \mathrm{~m}$

Fig. 3. PSD of QPSK spectrum with

Gaussian pulse-shaping filter, BT $=0.5$

applying to the double balanced mixers. Some studies have been done on base hand pulse-shaping and are described herein.

Many authors tried various pulse-shaping methods $[2,6]$. Several types of filters such as fifth-order Butterworth, third-order Bessel and Square Root Cosine are used. Pre-modulation pulse-shaping with different modulation schemes, such as Pulse Code Modulation(PCM), Binary Phase Shift Keying (BPSK), QPSK and Gaussian filtered Minimum Shift Keying(GMSK) have been studied in [2]. In this paper, a simple pre modulation filter has been employed to achieve thesecriteria. Here, we considereda Gaussian filter $(\mathrm{N}=5)$ and a new N5 pulse shaping filter and compared both. These pulse-shaping techniques are though applied at base band, but finally shifted its center frequency.

The QPSK signal is generated by linear addition of two quadrature BPSK signals $[4,7]$. Sharp transitions in the time domain lead to a relatively wide PSD that rolls off quite slowly. The first null occurs at a frequency equal to the data rate away 


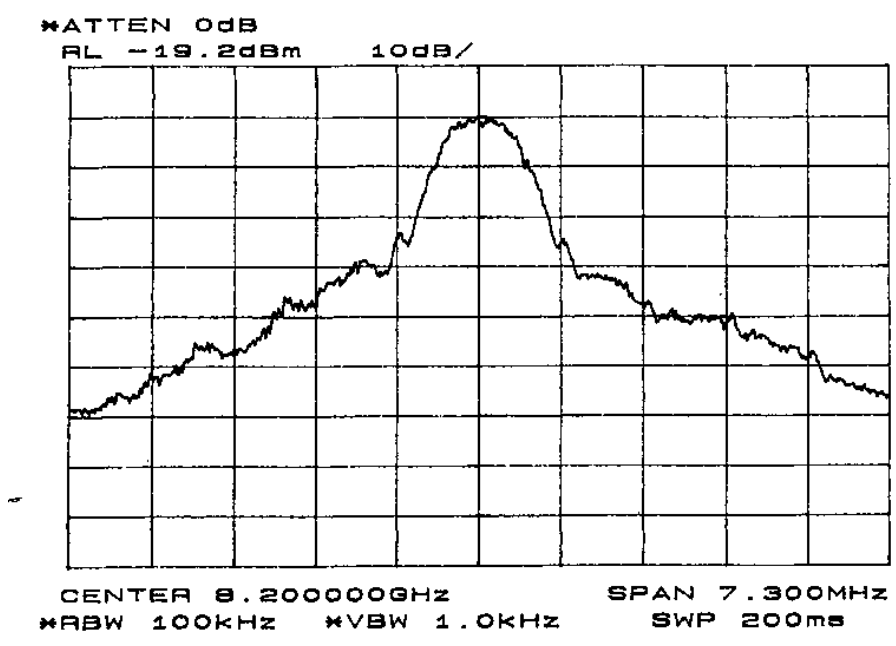

Fig. 4. PSD of QPSK spectrum with N5 pulse-shaping filter, $\mathrm{BT}=0.5$

from the carrier. The amplitude of the first lobe is only $13 \mathrm{~dB}$ down from its value at the centre frequency. It may be noted that $90 \%$ of the transmitted power of an unfiltered QPSK signal is within a bandwidth equal to the bit rate. Filtering the signal can greatly reduce the transmitted signal bandwidth.

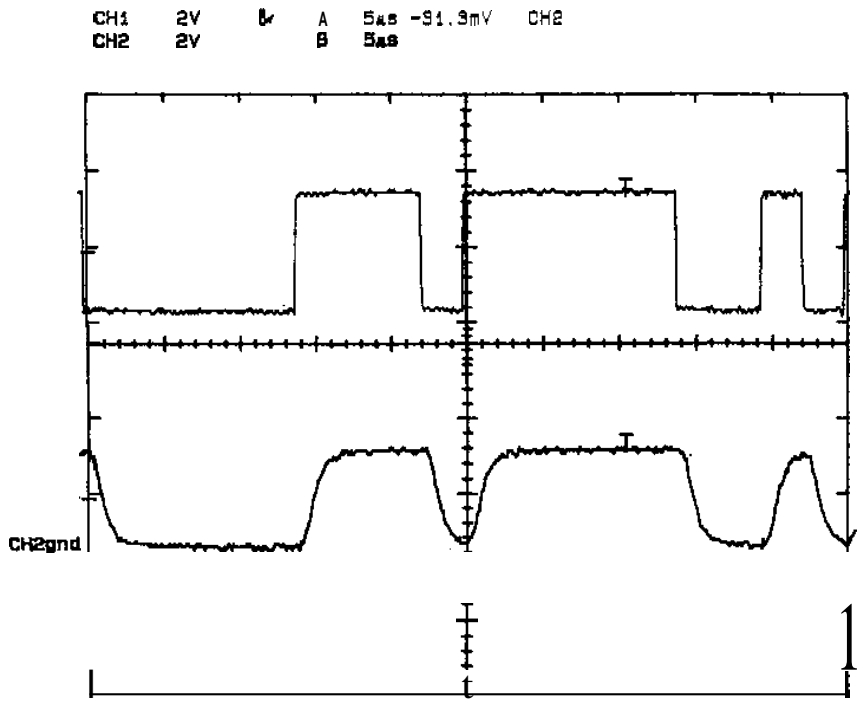

Fig. 5. Input data and shaped filtered output of Gaussianfilter

\section{HARDWARE IMPLEMENTATION}

An experiment was conducted to reduce the side lobe levels of PSK spectrum. The photograph of the hardware set up is shown in Figure 1. The experimental set-up block diagram is shown in Figure 2. A 3A, 3dB, 90 degrees coupler module of M/S Narda, 7-12 GHz, Model No. 4035C, Mixers of M/S Anaren, 8-12 GHz, Model No. 73128 used as double balanced mixers; and power combiner of MSS Narda, 2-8 GHz, Model
No. 4343 are used to make a QPSK modulator. The data was fed through a data generator of M/S Agilent Technologies, $H P$ 81200. The pulse-shaping circuit was designed on a breadboard. The NRZ data was passed through Gaussian filter and N5 pulse shaping circuit. The PSD of QPSK spectrum with Gaussian and N5 pulse shaping circuits are shown in Figures 3 and 4. From Figure 3, it may be observed that with Gaussian filter as pulse shaping circuit, the first and second side lobes are $-24 \mathrm{~dB}$ and $-35 \mathrm{~dB}$ with respect to the main lobe. Figure 4 shows the QPSK spectrum with the N5 pulse shaping circuit where the first and second side lobe levels are also $-32 \mathrm{~dB}$ and $-40 \mathrm{~dB}$ with respect to main lobe.

The $8.0 \mathrm{GHz}$ QPSK spectrum was down converted to 375 $\mathrm{MHz}$. The down converted signal was given to de-modulator. Figures 5 and 6 show the PRBS input data, shaped filter output data for Gaussian pulse shaping circuit, and N5 pulse shaping circuit, respectively. Figure 7 shows the input data and demodulated data of N5 pulse shaping filter. An observation shows that the demodulated data is identical to input shaped data except a small delay between input and output data. The performance can be improved for high bit rate applications by using a PCB with surface mountable components.

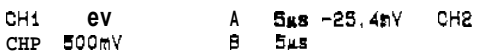

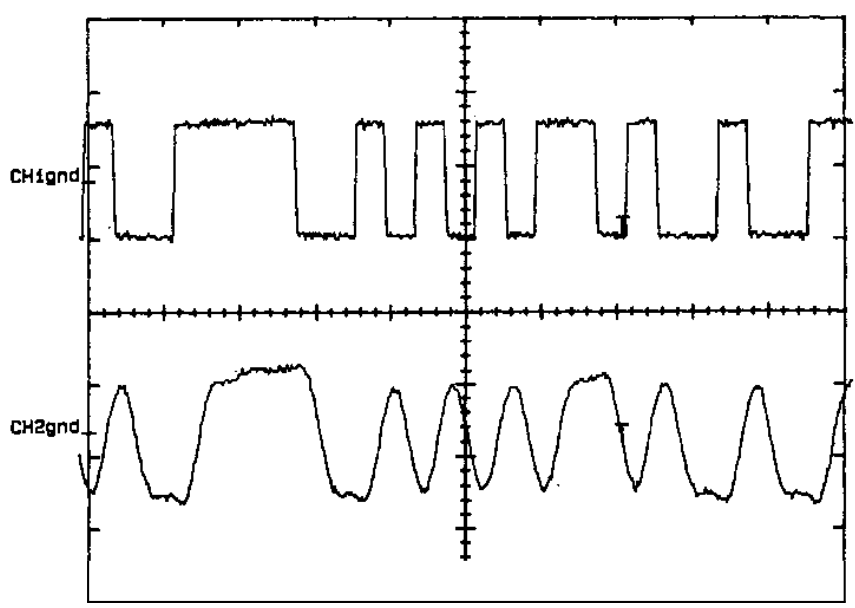

\section{Fig. 6. Input data and shaped filtered output of $\mathrm{N} 5$ pulse-shaping filter}

\section{SIDE LOBE LEVELS REDUCTION AND SPIKESELIMINATION}

At high bit rates, it is very difficultto realize a perfect square wave with zero rise time and fall time and also with data symmetry and data balance. If square wave is not ideal, then it is represented by continuous wave spectrum with discrete components at regular intervals. The discrete components are undesirable, since power is wasted in these components and also leads to interference. In actual spacecraft environment, it is very difficult to maintain perfect data balance at high bit rate data transmission, which results in spectral spikes at undesirable places. 


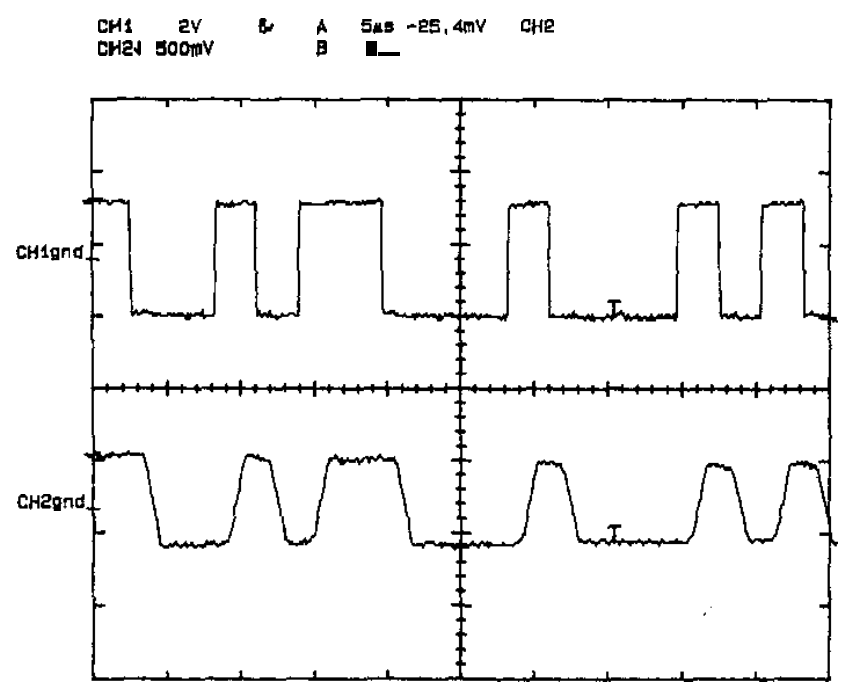

Fig. 7. Input data and demodulated output data of $\mathrm{N5}$ pulse shaping filter

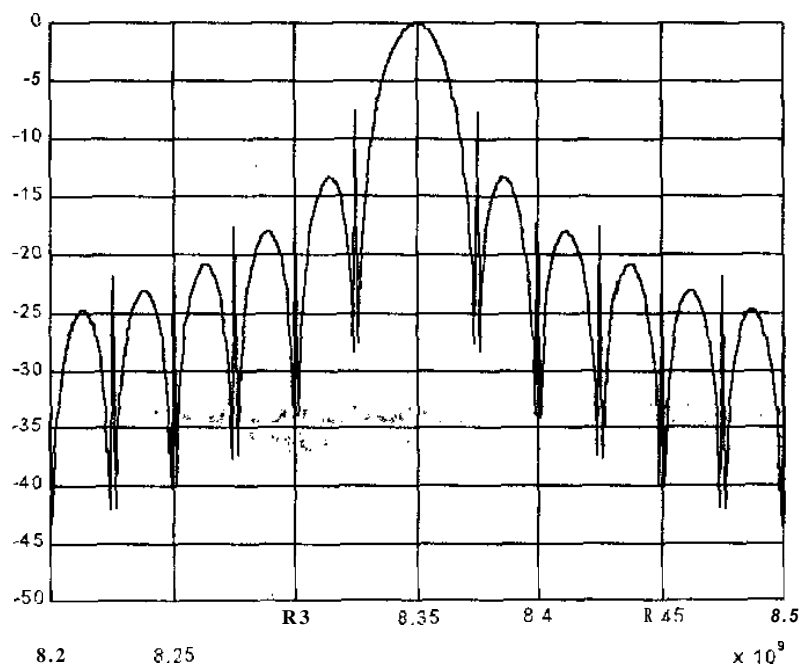

Fig. 8. Simulated PSD of QPSK spectrum with spikes

Ideal data has perfect symmetry with the time duration of a digit one to the time duration of a digit zero. It also has a perfect data balance such that the probability of getting a zero is equal to the probability of getting a one. In other words, $\operatorname{Pr}(0)=\operatorname{Pr}(1)$ $=0.5$ or $50 \%$. For non-ideal data, these two conditions, viz., data symmetry and data balance, are not possible. The data asymmetry can happen due to the stray capacitance in spacecraft wiring and the data imbalance can be produced by long runs of $1 \mathrm{~s}$ and $0 s$ in the random data. If non-ideal data are present (i.e., the mean value or expected value of the signal is not equal to zero) the PSD of the digital signal consists not only of a continuous spectrum that depends on the pulse shape spectrum of the signal data (rectangular pulse for NRZ-L), but also contains spectral lines (delta functions) spaced at approximately the harmonics of the symbol rate (RS).

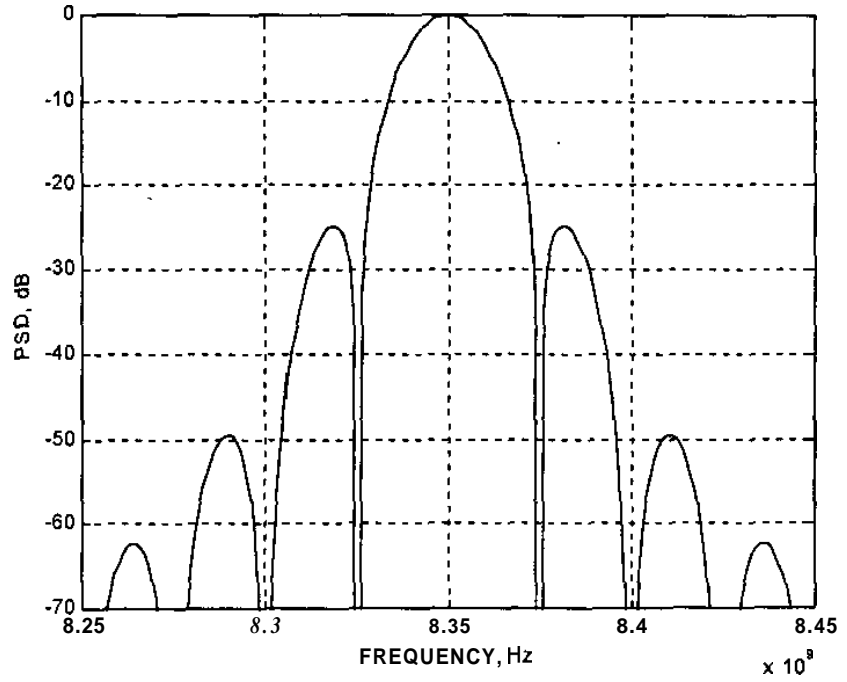

Fig. 9. Simulated PSD of proposed SS window function

The generation of such spectrum through the simulation of its PSD equation is little complicated. But such a spectrum can also be realized easily by suitably adding delta functions to QPSK spectrum at appropriate places. It is shown that this way one can simulate QPSK spectrum as shown in Figure 8, which will occur in non-ideal conditions. This spectrum is used for further study in this paper.

The new Spike Suppression (SS) Window proposed provides low side lobe levels also. The SS Window function equations in frequency domain and time domain are obtained and simulated PSD is shown in Figure 9. One can notice that the first and second sidelobe levels are $-25 \mathrm{~dB}$ and $-50 \mathrm{~dB}$, respectively. This reduction in sidelobe levels helps in reduing the interference with other systems.

The following section explains about spectral spikes that appear at high bit rate data transmission and elimination process. Many functions were simulated and studied but two functions were found suitable to remove spikes in QPSK spectrum.

\section{TRAPEZOIDAL WINDOW FUNCTION}

Digital signals with finite rise and fall times are well representedby a trapezoidal pulse [8]. Figure 8 represents PSD of QPSK spectrum with spikes. The resultant spectrum after the multiplication with trapezoidal function is given in Figure 10. It is found to have better characteristics such as low side lobe levels, which are very helpful in reducing interference between various systems. To further reduce the spike levels, a SS Window function is used. Several spectrums, apart from the trapezoidal spectrum, have been tried for spike removal. $S S$ Window function has been found to provide a better handling of spikes present in the QPSK spectrum compared to trapezoidal window function. This function not only reduces side lobe levels of QPSK spectrum but also useful for removing spikes. Figure 11 shows the QPSK spectrum with 
complete suppression of spikes. This window function finds suitability in digital signal processing based systems.

\section{CONCLUSION}

The need for reduction in QPSK spectrum side lobe levels is important to meet FCC regulations that would reduce the unwanted interference caused by spilling into adjacent channels. Pulse shaping technique is being used here to reduce the bandwidth of the spectrum. A simple filter at the input provides the desired effect. The incorporation of this filter with the hardware has provided the required effect as shown in the figures.Practicality of such a pre modulation filter for reducing side lobe levels has been demonstrated and is expected to be used in future GSAT mission applications. Also, new SS Window function has been suggested which helps in reducing the side lobe levels. It also avoids the interference with near by frequencies.

Practical characterization of square wave characteristicsis very difficult in reality due to high bit rate data transmission. Some ideas to achieve ideal spectrum without spectral spikes have been present in this paper. Observations clearly demonstrate that the SS Window function provides superior characteristics suitable for high bit rate data transmission particularly for space communications.

\section{REFERENCES}

[1] Caballero, R, 1996,

8.PSK signaling over non-linear satellite channels, NTIS report - 1996.

(2) Oelting, J. D., May 1993-1995,

CCSDC.SPCG Efficient Modulation Study, A comparison of Modulation Schemes,

Phase 1-3, May 1993-1995.

[3] Stanley, W.D.1,975,

Digital SignalProcessing,

Reston Publishing Company, LDc.,

A Prentice Hall Company, Reston, Virginia, 1975.

[4] Sambasiva, Rao V., Ramana, D.V.and Pal, S., March-April 1999, Some experimentationon X-band Offset QPSK modulator, [ETE Technical Review, Vol. 16, No. 2, March-April, 1999.

[5] Ramana, D.V. and Rao, K.N.S., September-December 1999, Effects of TWTA on the bandlimited QPSK signals, IETE Sourna! of Research, Vol. 45, Septeriber-December 1999.

[6]. Mania, W.L., et al., 1997,

Efficient Modulation methods study at NASA/JPL SFCG meeting. Galveston, Texas. 1997.

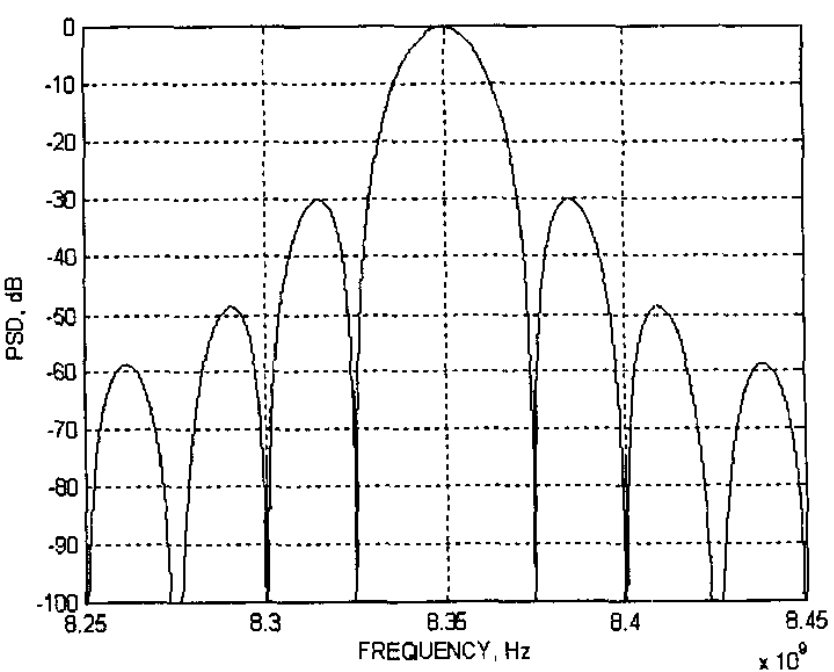

Fig. 10. Removal of spikes by trapezoidal window function

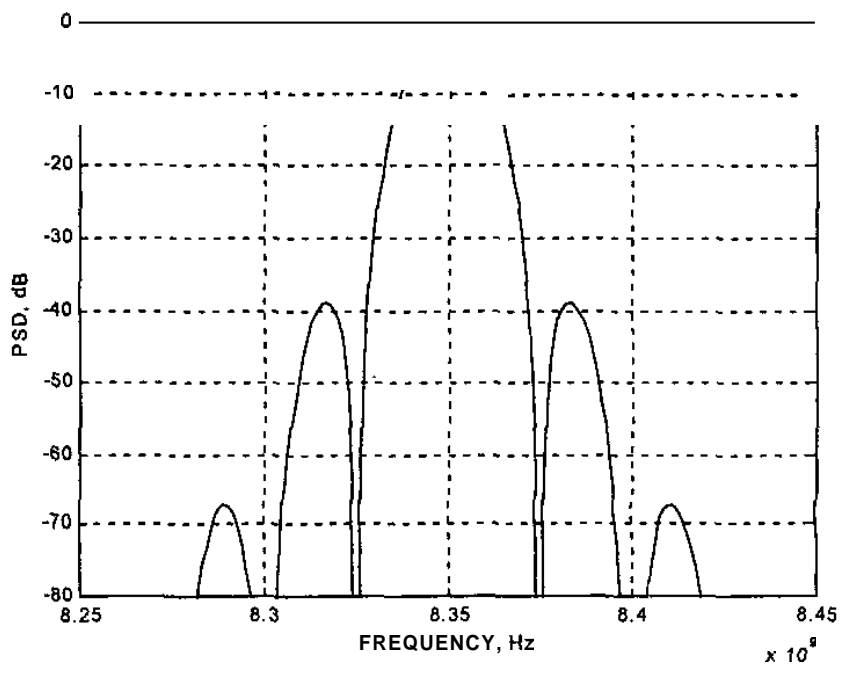

Fig. 11. Spikes suppressionby using SS window function

[7] Larson, L.E.,

$\mathrm{RF}$ and Microwave circuit design for wireless communications, Artech House, Boston, London.

[8] Lathi, B.P., 1993,

Moderm digital and Analog Communication Systems, Prism books Pvt. Ltd., 1993. 